\title{
Microstructural strain energy of $\alpha$-uranium determined by calorimetry and neutron diffractometry
}

\author{
M. E. Manley, ${ }^{1,2}$ B. Fultz, ${ }^{1}$ D. W. Brown, ${ }^{2}$ B. Clausen, ${ }^{1,2}$ A. C. Lawson, ${ }^{2}$ J. C. Cooley, ${ }^{2}$ W. L. Hults, ${ }^{2}$ \\ R. J. Hanrahan, Jr., ${ }^{2}$ J. L. Smith, ${ }^{2}$ and D. J. Thoma ${ }^{2}$ \\ ${ }^{1}$ California Institute of Technology, Pasadena, California 91125 \\ ${ }^{2}$ Los Alamos National Laboratory, Los Alamos, New Mexico 87545
}

(Received 8 January 2002; revised manuscript received 22 April 2002; published 22 July 2002)

\begin{abstract}
The microstructural contribution to the heat capacity of $\alpha$-uranium was determined by measuring the heatcapacity difference between polycrystalline and single-crystal samples from 77 to $320 \mathrm{~K}$. When cooled to 77 $\mathrm{K}$ and then heated to about $280 \mathrm{~K}$, the uranium microstructure released $(3 \pm 1) \mathrm{J} / \mathrm{mol}$ of strain energy. On further heating to $300 \mathrm{~K}$, the microstructure absorbed energy as it began to redevelop microstrains. Anisotropic strain-broadening parameters were extracted from neutron-diffraction measurements on polycrystals. Combining the strain-broadening parameters with anisotropic elastic constants from the literature, the microstructural strain energy is predicted in the two limiting cases of statistically isotropic stress and statistically isotropic strain. The result calculated in the limit of statistically isotropic stress was $(3.7 \pm 0.5) \mathrm{J} / \mathrm{mol} \mathrm{K}$ at $77 \mathrm{~K}$ and $(1 \pm 0.5) \mathrm{J} / \mathrm{mol}$ at room temperature. In the limit of statistically isotropic strain, the values were $(7.8$ $\pm 0.5) \mathrm{J} / \mathrm{mol} \mathrm{K}$ at $77 \mathrm{~K}$ and $(4.5 \pm 0.5) \mathrm{J} / \mathrm{mol}$ at room temperature. In both cases the changes in the microstructural strain energy showed good agreement with the calorimetry.
\end{abstract}

DOI: 10.1103/PhysRevB.66.024117

PACS number(s): 81.30.-t, 83.85.Hf

\section{INTRODUCTION}

We report direct measurements of the microstructural effects on the heat capacity of $\alpha$-uranium. Results from prior work imply that microstructure can affect the charge-densitywave (CDW) transitions in $\alpha$-uranium. Distinct CDW transitions at 23,37 , and $41 \mathrm{~K}$ can be seen clearly in calorimetry measurements on single-crystal uranium, but these transitions are either suppressed or too severely broadened to be observed in measurements on polycrystalline samples. ${ }^{1}$ Work by Hall ${ }^{2}$ shows that the constraints on anisotropic thermal expansion of uranium crystallites in polycrystals either partially inhibit or prevent the CDW transformations, as evidenced by the diminished CDW effects on thermalexpansion, heat-capacity, and electron-transport properties.

Recently it has been proposed that a significant portion of the anharmonic contribution to heat capacity of $\mathrm{Ni}_{3} \mathrm{~V}$ and $\mathrm{Pd}_{3} \mathrm{~V}$ alloys was the result of strains in the microstructure. ${ }^{3,4}$ In these experiments, however, the microstructural contribution was mixed with contributions from both harmonic phonons and anharmonic volume expansion, which are difficult to separate. The phonon contribution is an especially difficult problem in the case of uranium because of an unusually large thermal softening of its phonon density of states. ${ }^{5}$ In the present work, the microstructural contribution in uranium is isolated by measuring the difference between the specific heat of a polycrystal and a mass-matched single crystal. In addition, neutron powder-diffraction experiments are used to measure the distribution of elastic strains in the polycrystalline material along various crystallographic directions. Neutrons rather than $\mathrm{x}$ rays were used because of their ability to penetrate and give a true volume average. The strain distribution data are used to calculate the microstructural strain energy. We compare the elastic strains that develop in polycrystalline $\alpha$-uranium on heating to the reduc- tion in the measured heat capacity as this energy is released. We show that the temperature and energy scales of these phenomena are similar to the measured distortions of the CDW transitions in polycrystalline $\alpha$-uranium.

\section{EXPERIMENT}

Uranium crystals were grown by electrotransport through a molten salt bath of LiCI-KCl eutectic containing approximately $3 \mathrm{wt} \% \mathrm{UCl}_{3} .{ }^{6}$ Uranium grew on a stainless steel cathode as dendrites shaped as parallelogram-edged platelets. The platelets are high-purity single crystals of $\alpha$-uranium. The residual resistivity ratio (RRR) of 115 was about three times higher than any RRR reported previously. ${ }^{1}$ Because the uranium was deposited below the $\alpha$ - $\beta$ transformation temperature, single crystals are strain free. Strips were cut by spark-erosion cutting, and were cleaned in concentrated $\mathrm{HNO}_{3}$ and electropolished in $\mathrm{H}_{3} \mathrm{PO}_{4}$.

Uranium polycrystals were prepared by induction melting the dendritic electrorefined product described above in a $\mathrm{BeO}$ crucible under an inert atmosphere. The ingot was melted only once to minimize the risk of contamination from the crucible or the atmosphere. The samples were sectioned directly from the cast pancake shaped ingot with a diamond saw so that the long axis of the sample was along a radius.

Differential heat-capacity measurements were performed with a Perkin-Elmer DSC-4 differential scanning calorimeter (DSC) that had been modified by installing its sample head in a liquid-helium dewar. ${ }^{7}$ Mass-matched 100-mg samples, one single crystalline and one polycrystalline, were placed in the two sample pans of the DSC. Heat-capacity measurements comprised pairs of runs, with the two samples interchanged in the sample pans between runs. The difference in heat capacity was found from the difference of these two sets of runs by a procedure whereby the background is corrected and the signal is doubled. Because the samples had the same chemical composition and the same mass, the measured sig- 


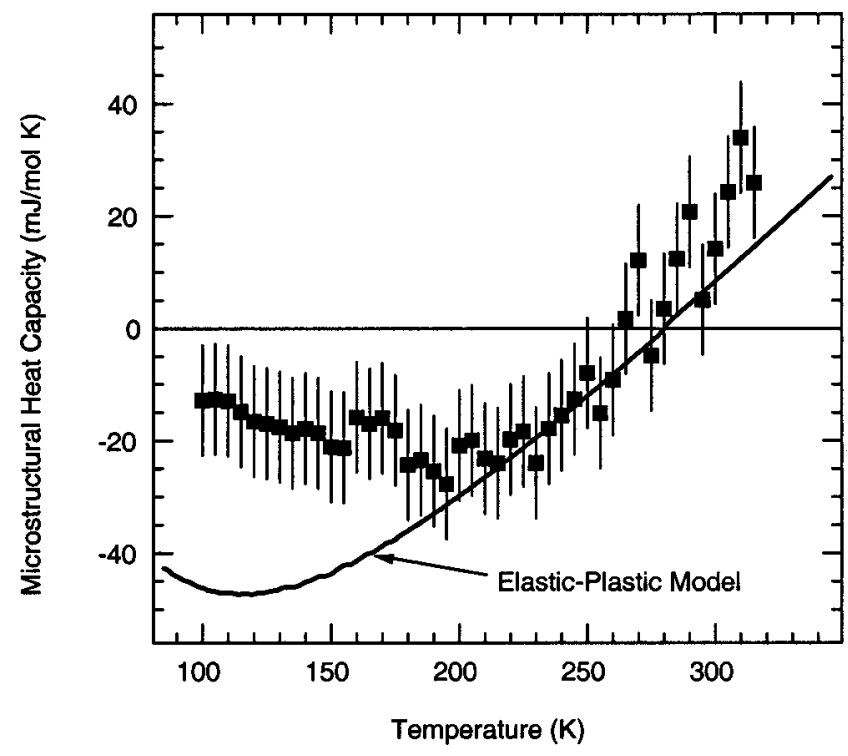

FIG. 1. Microstructural contribution to the specific heat of uranium. The elastic-plastic model, described by Manley et al., ${ }^{4}$ was scaled vertically and a Debye temperature of $250 \mathrm{~K}$ was used. The error bars are the standard deviation between an average of four pairs of runs.

nal is the microstructural effect on the heat capacity of the polycrystalline sample. Four matched runs were performed to ensure reproducibility. To counteract instrumental drift, runs comprised two pairs of scans over temperature intervals of $30 \mathrm{~K}$, which overlapped by $10 \mathrm{~K}$.

Neutron-diffraction patterns were obtained on the Neutron Powder Diffractometer (NPD) at the Manuel Lujan, Jr. Neutron Scattering Center, Los Alamos National Laboratory. The 150 -g sample was cut from the same material as the polycrystal used in the calorimetry. The sample was oriented so that its long axis (the radial direction in original ingot) was perpendicular to the scattering plane, and the faces (cut perpendicular to the tangent of the round ingot) were at $45^{\circ}$ with respect to the incident beam. To reproduce the thermal history of the calorimetry measurements the sample was first cooled to $77 \mathrm{~K}$. Diffraction patterns were then obtained at 77 and $90 \mathrm{~K}$, and in steps of $10 \mathrm{~K}$ up to $290 \mathrm{~K}$. The sample was equilibrated at each temperature for $10 \mathrm{~min}$ before acquiring the diffraction pattern. Each diffraction pattern was acquired for $20 \mathrm{~min}$. The sample was re-cooled to check for irreversibilities, and measurements were then performed at lower temperatures $(40,30$, and $20 \mathrm{~K})$.

\section{RESULTS AND ANALYSIS}

The crystallographic anisotropy of thermal expansion in uranium is expected to cause a buildup of elastic strains as a polycrystalline sample is heated or cooled to temperatures different from $T_{0}$, the temperature where the strain energy is a minimum. The shape of the microstructural contribution to the heat capacity showed the form expected from the "elastic-plastic model" described by Manley et al., ${ }^{4}$ Fig. 1. Briefly, the model treats the strains as elastic as the material is reheated with the condition that $T_{0}$ changes when changes in temperature exceed a limit for internal yielding, $\Delta T_{y}$. The polycrystalline sample showed the release of microstructural strain energy upon heating from liquid-nitrogen temperature. The heat capacity crosses zero at about $280 \mathrm{~K}$ so we use this temperature as $T_{0}$. The estimated temperature change associated with yielding, as defined by Manley et al., ${ }^{4}$ is thus $\Delta T_{y}=200 \mathrm{~K}\left(\Delta T_{y} \approx T_{0}-T_{\text {min }}\right.$ after cooling to a minimum temperature $\left.T_{\min }\right)$. Both of these values are much larger than for polycrystalline samples of tetragonal $\mathrm{DO}_{22}$ phase $\mathrm{Pd}_{3} \mathrm{~V},{ }^{4}$ reflecting the higher strength of uranium metal. The lowtemperature range, however, showed a significant difference from the elastic-plastic model. With constant thermal and elastic properties, the model predicts linear behavior until the material is cooled below about half the Debye temperature (approximately $150 \mathrm{~K}$ for uranium), where the thermalexpansion coefficients decrease owing to a depopulation of phonons. The data, however, show nonlinear behavior at much higher temperatures. This is probably a result of the strong temperature dependence of the elastic properties of uranium. A more detailed analysis, including the temperature dependence of the thermal and elastic properties, is discussed below.

The strain energy per unit volume in an arbitrary stress state is given in matrix notation by ${ }^{8}$

$$
u=\frac{1}{2} c_{i j} \varepsilon_{i} \varepsilon_{j} \quad(i, j=1 \cdots 6),
$$

where $c_{i j}$ is the stiffness matrix and $\varepsilon_{i}$ is the strain matrix. The total energy per unit volume in a polycrystal is obtained by averaging over the entire volume. Neutron diffraction provides a sampling of the strain distribution in crystallites in specific orientations determined by Bragg's law. We assume that the $\varepsilon_{i}$ in the set of all crystallites in orientations selected by Bragg's law and projected into crystallographic coordinates have a Gaussian distribution characterized by

$$
\delta_{i j}^{2}=\left\langle\varepsilon_{i} \varepsilon_{j}\right\rangle^{*}-\left\langle\varepsilon_{i}\right\rangle^{*}\left\langle\varepsilon_{j}\right\rangle^{*}
$$

where $\delta_{i i}^{2}=\sigma^{2}\left(\varepsilon_{i}\right)$, the variance of $\varepsilon_{i}$, and $\langle x\rangle^{*}$ gives the average of quantity $x$ in the crystallites selected by the diffraction condition. Since the crystallites in this calculation are all projected into the same coordinates we can multiply Eq. (2) by $c_{i j} / 2$, consider the appropriate sums, and rearrange terms to obtain an expression for the average strain energy [Eq. (1)] for the selected crystallites,

$$
\langle u\rangle^{*}=\left\langle\frac{1}{2} c_{i j} \varepsilon_{i} \varepsilon_{j}\right\rangle^{*}=\frac{1}{2}\left[\left\langle\sigma_{j}\right\rangle^{*}\left\langle\varepsilon_{j}\right\rangle^{*}+c_{i j} \delta_{i j}^{2}\right]
$$

where the relation between the stress and strain matrix, $\sigma_{j}$ $=c_{i j} \varepsilon_{i}$, has been used. The first term in Eq. (3) originates with the average distortion, and the second term originates with deviations from the average. For a polycrystal with random crystallite orientations, the average of one set of crystallites in a specific orientation is equivalent to any other and hence is equivalent to a volume average. An important con- 
sequence of the equivalence of a volume and powder average is that for static equilibrium, the stress tensor averaged over the entire volume must vanish, i.e., $\left\langle\sigma_{j}\right\rangle=0$. Thus for a powder average the first term in Eq. (3) is zero and the microstructural strain energy can be reduced to

$$
\langle u\rangle_{\mu \mathrm{str}}=\frac{1}{2} c_{i j} \delta_{i j}^{2} .
$$

Like the stiffness tensor, the strain-broadening tensor is fourth rank because it connects two second-rank tensors.

Because we need to know only the deviations from the average strain to determine the microstructural strain energy, we have to consider only the strain broadening in the neutron-diffraction data. This is an important simplification because the average strains can be determined accurately only with precise knowledge of the free crystal lattice parameters, which are often sensitive to impurities, defect concentrations, etc.

A measure of the strain-broadening matrix can be extracted from neutron-diffraction data using a formalism developed by P. W. Stephens. ${ }^{9}$ In this formalism the variance of the diffraction peak widths are fit using ${ }^{9}$

$$
\sigma^{2}(h k l)=\sum_{H K L} S_{H K L} h^{H} k^{K} l^{L},
$$

where the coefficients $S_{H K L}$ are restricted by symmetry (6 for orthorhombic) and $h, k$, and $l$ are the Miller indices. In the following discussion we convert this into strain using

$$
S(h k l)=\frac{\sqrt{\sigma^{2}(h k l)}}{d(h k l) C_{\mathrm{dif}}},
$$

where $d(h k l)$ is the spacing of $(h k l)$ planes and $C_{\text {dif }}$ is the diffraction constant that converts time-of-flight to $d$ spacing. The Stephens formalism has been incorporated into the wellknown GSAS (General Structure and Analysis Software) Rietveld refinement package. ${ }^{10}$ Strain broadening was determined by refining all neutron-diffraction data in the Le Bail mode. ${ }^{11}$ In this mode the diffraction peak intensities are treated as free parameters. Only the peak positions and profiles are fit since all the information on strain is contained in these parameters. Typical fits are shown in Fig. 2. Using profile function 4 in the GSAS software, the appropriate Stephens strain-broadening parameters (6 unique $S_{H K L}$ ) were extracted at each temperature. Of the four detector banks $\left( \pm 90^{\circ}, \pm 148^{\circ}\right)$, only the two high-resolution banks $\left( \pm 148^{\circ}\right)$ gave meaningful results. From the strain-broadening parameters, the microstrain broadening was calculated as a function of crystallographic direction. The function $S(h k l)$ fits simultaneously the strain broadening in all of the peaks. To check for consistency, the peak broadening from this function was compared to single-peak fits for some easily separated peaks, Fig. 3. The fair agreement was reassuring, although it fell short of what Stephens obtained using much higher resolution synchrotron radiation. ${ }^{9}$ The temperature trend of a single peak, shown in the inset of Fig. 3, was much better.

Three-dimensional representations of the strain broadening function at three temperatures are shown in Fig. 4 for both high-resolution detector banks. There are some differences between the microstrain distributions sampled by the two banks. This could be caused by the crystallographic texture in the sample. A simultaneous refinement of peak intensities for all four banks gave a texture index of 2.5. ${ }^{10}$ The most significant difference is that the microstrains in the [010] direction observed by bank 1 are much larger than those observed in bank 2 . In both cases the strain broadening is strongly anisotropic, as is expected from the anisotropic elastic and thermal-expansion properties of $\alpha$-uranium. ${ }^{1,12}$ On average the largest strain broadening is in the [010] direction while the [001] and [100] directions are similar. This distribution of microstrains at $290 \mathrm{~K}$ reflects the symmetry of the uniaxial compliance (inverse Young's modulus; $E_{100}^{-1}$ $=4.91 \mathrm{GPa}^{-1}, E_{001}^{-1}=4.88 \mathrm{GPa}^{-1}, E_{010}^{-1}=6.74 \mathrm{GPa}^{-1}$ ) rather than the hydrostatic compressibility $\left(\beta_{100}=3.80 \mathrm{GPa}^{-1}\right.$, $\left.\beta_{001}=2.25 \mathrm{GPa}^{-1}, \quad \beta_{010}=2.92 \mathrm{GPa}^{-1}\right){ }^{1,12}$ Thus the observed distribution of microstrains shows that the average crystallite is in a stress state closer to uniaxial than hydrostatic. The magnitude of the strain broadening decreases with increasing temperature. This agrees with the microstructural specific heat. The microstructural specific heat is negative at low temperatures, indicative of a decrease in strain energy with increasing temperature.

\section{MICROSTRUCTURAL STRAIN ENERGY}

To determine the strain energy stored in the microstructure, we need to determine the strain-broadening matrix elements $\delta_{i j}^{2}$, corresponding to the nine stiffness constants $c_{i j}$, allowed by orthorhombic symmetry. The first six components correspond to the variance of the three pure normal strains and the three pure shear strains. They can be written down directly as

$$
\begin{aligned}
& \delta_{11}^{2}=S^{2}(100), \\
& \delta_{22}^{2}=S^{2}(010), \\
& \delta_{33}^{2}=S^{2}(001), \\
& \delta_{44}^{2}=S^{2}(011), \\
& \delta_{55}^{2}=S^{2}(101), \\
& \delta_{66}^{2}=S^{2}(110),
\end{aligned}
$$

where the numbers in parentheses correspond to the crystallographic $h k l$ indices. The powder average microstrains [Eq. (8)] are approximated by averaging the results of banks 1 and 

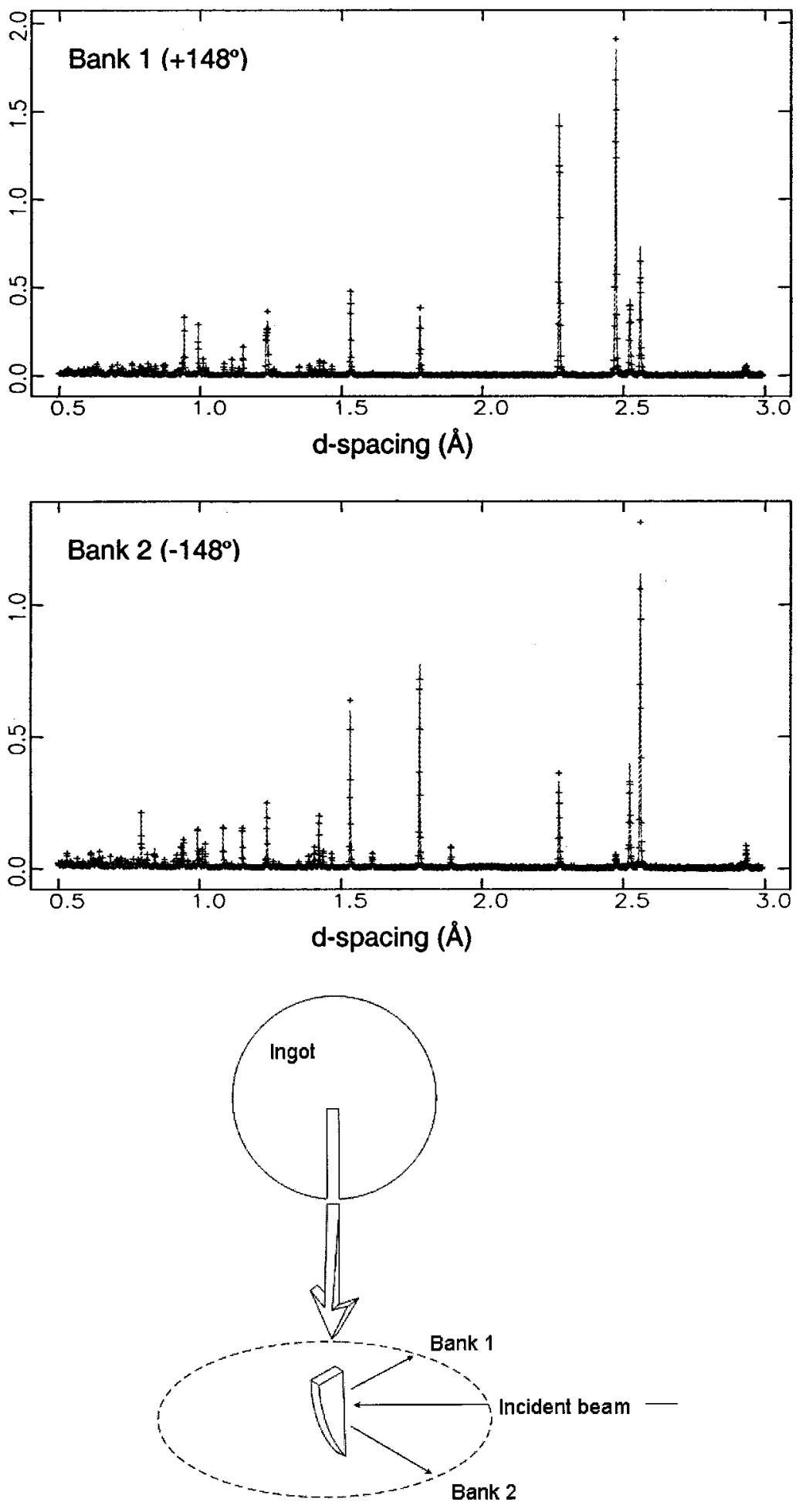

2 (Fig. 4). The remaining nonzero components (corresponding to $\left.c_{12}, c_{13}, c_{23}\right)$ contain subscripts with $i \neq j$ and thus represent connected variances between strain components [Eq. (2)]. The six coefficients obtained in the Stephens formalism cannot be used to determine these strain components. However, these strain components can be estimated.

Each connected variance term describes a correlation between strain components in different directions, averaged over the volume of the sample. To approximate this contri-
FIG. 2. Refinement of uranium diffraction patterns in Le Bail mode at $190 \mathrm{~K}$ from detector banks $1\left(+148^{\circ}\right)$ and $2\left(-148^{\circ}\right)$. Intensities are in arbitrary units. The orientation of the detector banks with respect to the scattering and sample geometry is shown at the bottom. Pancake shaped ingot is viewed from above with sample cut away. bution two limits can be considered: (1) statistically isotropic strains and (2) statistically isotropic stresses. In the case of statistically isotropic strains, there are no correlations so this contribution is zero. For statistically isotropic stresses, the strain in direction 1 due to the stress in direction 1 is uncorrelated with the strain in direction 2 due to the stress in direction 2, and thus makes no contribution to the connected variance term. However, the strain in direction 1 due to the stress in direction 2 (determined by the Poisson ratio $\nu_{12}$ ) is 
correlated with the strain in direction 2 due to the stress in direction 2 and thus contributes to $\delta_{12}^{2}$. Therefore assuming statistically isotropic stresses the only correlations between strains in different directions comes from the Poisson effect. The connected variance terms in the strain-broadening matrix are thus

$$
\delta_{i j}^{2}=\left\{\begin{array}{cc}
0 & \text { isotropic strains } \\
\left\langle\varepsilon_{i} \varepsilon_{j}\right\rangle-\left\langle\varepsilon_{i}\right\rangle\left\langle\varepsilon_{j}\right\rangle=-\nu_{i j}\left[\left\langle\varepsilon_{j}^{2}\right\rangle-\left\langle\varepsilon_{j}\right\rangle^{2}\right]=-\nu_{i j} \delta_{j j}^{2} & \text { isotropic stresses }
\end{array}\right.
$$

for $i \neq j$. As pointed out by Stokes and Wilson, ${ }^{13}$ who assume statistically isotropic stresses, the true stress-strain distribution is likely to be somewhere in between these limits.

Fisher and McSkimin ${ }^{14}$ measured all of the single-crystal elastic constants of $\alpha$-uranium at room temperature. Some properties are most unusual. For example, there is an extraordinarily strong coupling between strains in the [010] direction and the [001] direction with $\nu_{32}=0.548$. On the other hand, the strains along the [100] direction and the [001] direction are almost uncoupled with $\nu_{31}=-0.017$. It is therefore important that the coupling terms, Eq. (8), be included when calculating the strain energy. Fisher ${ }^{12}$ measured the temperature dependence of the shear and normal compliance coefficients. The temperature dependencies of the offdiagonal components $\left(c_{12}, c_{13}\right.$, and $\left.c_{23}\right)$ are unknown, so their temperature dependencies were neglected. Substituting Eqs. (7) and (8) into Eq. (4) with the temperature dependent elastic constants, the strain energy stored in the microstructure was calculated as a function of temperature for both cases of statistically isotropic stress and statistically isotropic strains, Fig. 5.

For comparison, the microstructural specific heat shown in Fig. 1 was integrated to give a measure of the strain energy using

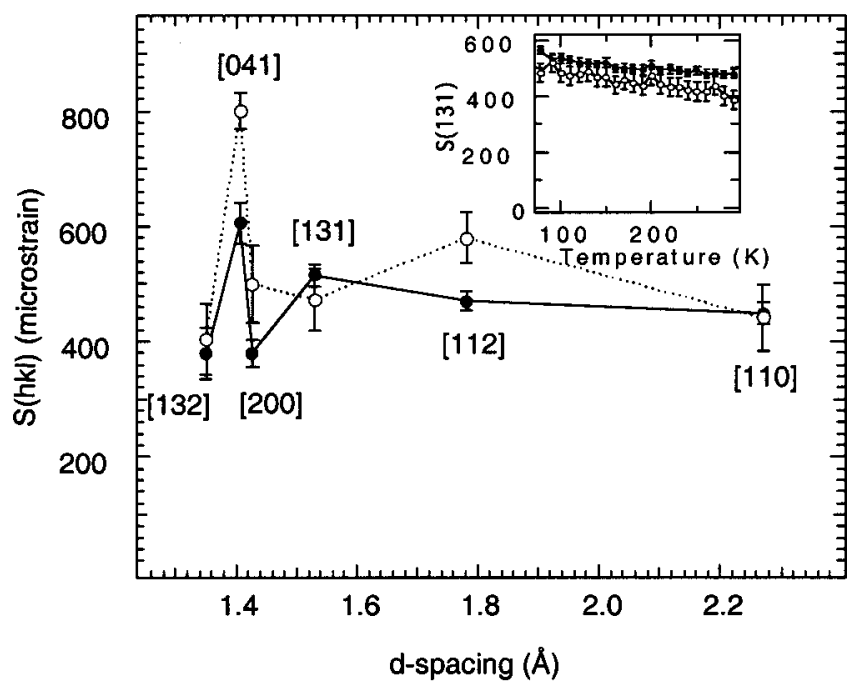

FIG. 3. Comparison of strain broadening at $190 \mathrm{~K}$ seen in bank $1\left(+148^{\circ}\right)$ calculated from refinement of the entire diffraction pattern, open symbols $(\bigcirc)$, and from single-peak fits, filled symbols (O). The inset shows the temperature dependence of one peak. Units are microstrain $(1000=0.1 \%$ strain $)$.

$$
E_{\mu \mathrm{str}}=\int C_{\mu \mathrm{str}}(T) d T+E_{0},
$$

where $E_{0}$ is an arbitrary constant set to match the strain energy determined from the strain broadening in both limits, Fig. 5. The origin of $E_{0}$ is probably residual strain fields from both macroscopic strains in the microstructure as well as from microscopic strains near crystal defects (dislocations, vacancies, etc.). The agreement in Fig. 5 between the neutron-diffraction and calorimetry results gives more credibility to both measurements. Although it appears that the statistically isotropic strain limit is a better fit to the calorimetry result, the discrepancy in the statistically isotropic stress limit could come from the fact that the temperature dependence of the off-diagonal stiffness constants is not known.

Errors in the total energy integral are not shown in Fig. 5 because they depend on the uncertainty in $E_{0}$ and the systematic accumulation of errors through integration. For example, if $E_{0}$ is assumed known exactly at some temperature, say $T_{0}$, then the error in energy by integrating away from $T_{0}$ is given by $\Delta E_{\mu \text { str }}=\Delta C_{\mu \text { str }}\left(T-T_{0}\right)$ where $\Delta C_{\mu \text { str }}$, the error in the specific heat measurement, is $0.01 \mathrm{~J} / \mathrm{mol} \mathrm{K}$. Thus considering the statistically isotropic stress limit, assuming $E_{0}$ $=1 \mathrm{~J} / \mathrm{mol} \mathrm{K}$ at $280 \mathrm{~K}$, then the energy at $77 \mathrm{~K}$ would be $(4$ $\pm 2) \mathrm{J} / \mathrm{mol} \mathrm{K}$. The strain energy calculated from the diffraction measurements in the statistically isotropic stress limit give a more precise measure at $77 \mathrm{~K}$ of $(3.7$ $\pm 0.5) \mathrm{J} / \mathrm{mol} \mathrm{K}$. In the statistically isotropic strain limit, $E_{0}$ $=4.3 \mathrm{~J} / \mathrm{mol} \mathrm{K}$ at $280 \mathrm{~K}$ and the strain energy at $77 \mathrm{~K}$ is (7.8 \pm 0.5$) \mathrm{J} / \mathrm{mol} \mathrm{K}$.

Attempts were made to measure how the strain energy stored in the microstructure changed below the CDW transitions by measuring the diffraction pattern at 40,30 , and 20 K. However, there was no clear indication that a CDW transition had occurred. Specifically, in single-crystal samples Barrett et al. ${ }^{15}$ observed a sudden increase in the $a$ and $b$ lattice parameters by $0.2 \%$ and $0.05 \%$, respectively, and a decrease in $c$ of $-0.09 \%$. Our measurements, on the other hand, showed no significant changes other than the usual continuous thermal contractions. Our results agree with earlier measurements suggesting that CDW transformations are either suppressed or completely smeared out in temperature in the presence of the microstructural constraints on the anisotropic thermal expansions or contractions. ${ }^{1,2}$ 

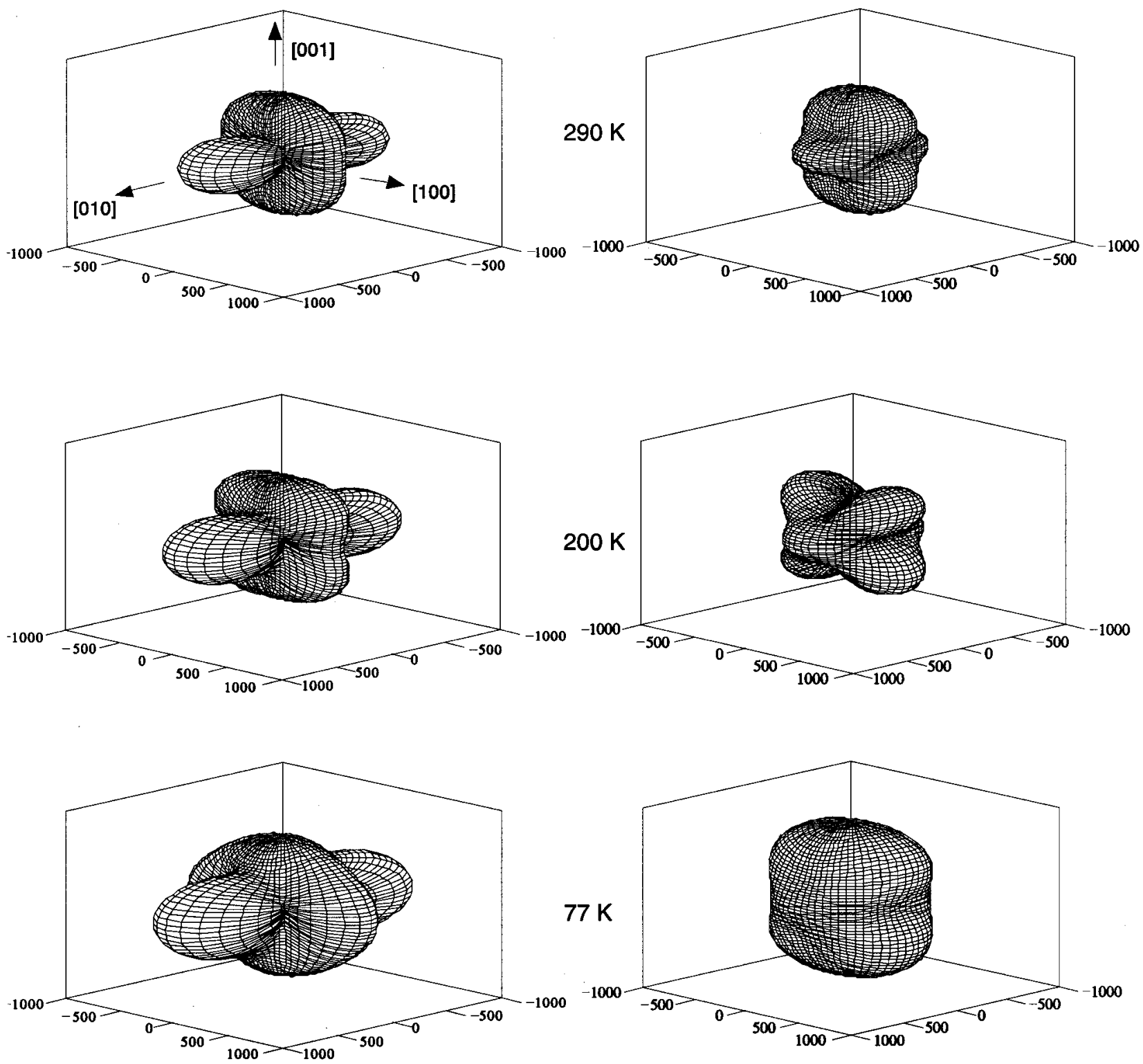

Bank $1\left(+148^{\circ}\right)$

Bank $2\left(-148^{\circ}\right)$

FIG. 4. These shapes represent the anisotropic microstrain broadening in uranium at several temperatures measured from the two high-resolution detector banks. Units are microstrain $(1000=0.1 \%$ strain $)$, and all axes are on the same scale.

\section{ELASTIC STRAINS AND CHARGE-DENSITY-WAVE TRANSITIONS}

The amount of increase of strain energy in the microstructure on cooling, about $3 \mathrm{~J} / \mathrm{mol} \mathrm{K}$ at $77 \mathrm{~K}$ in either limit (Fig. 5 ), is comparable to the latent heats of the CDW transitions. The latent heats of the transitions at 37 and $22 \mathrm{~K}$ are 2.08 and $1.38 \mathrm{~J} / \mathrm{mol}$, respectively. ${ }^{16,17}$ Since these transitions are accompanied by lattice strains, it is therefore not surprising that the transitions are either smeared out in temperature or suppressed by constraints imposed by the microstructure.

The expansion of the $a$ axis and $b$ axis during the CDW transitions ${ }^{15,18}$ would tend to undo some of the strains that develop during cooling because these strains are contractions along these directions. Specifically, in single crystals the strains of the CDW transition cause the lattice parameters to recover their values at about $180 \mathrm{~K}$ for the $a$ axis and at about $300 \mathrm{~K}$ for the $b$ axis. ${ }^{15,18}$ Therefore one would expect that in regions dominated by thermal strains along [100] and [010], the strain energy would favor the transitions. These regions in polycrystalline material may undergo CDW transitions at higher temperatures than in single crystals. On the other hand, the contraction of the $c$ lattice parameter during the transition would tend to further increase the strain energy. The opposite effect may therefore be expected in crystallites dominated by strains along [001]. In a polycrystal with randomly oriented crystallites, we would expect a distribution of 


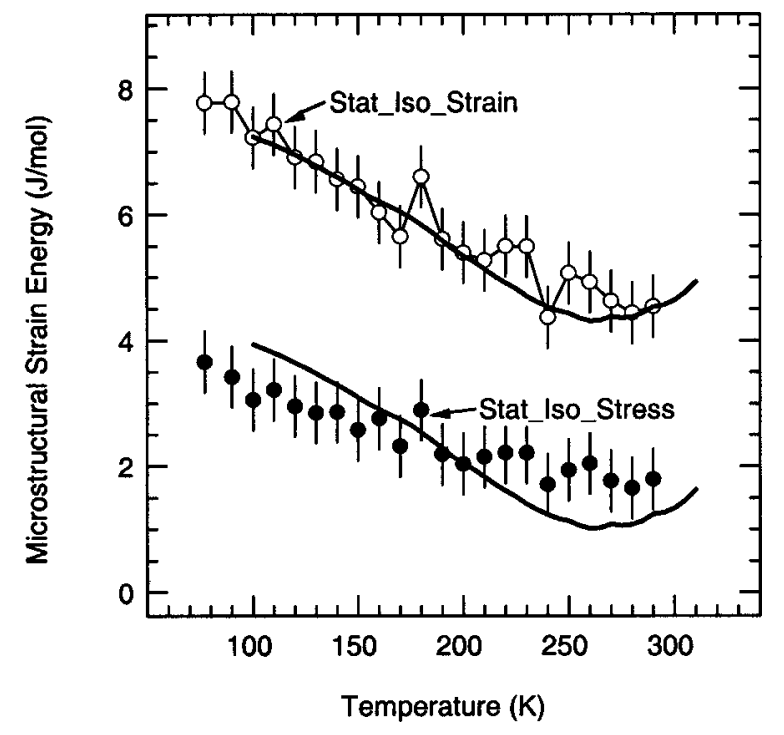

FIG. 5. Strain energy stored in the microstructure: The solid circles $(\bigcirc)$ and the open circles $(\bigcirc)$ were calculated from strain broadening in the statistically isotropic stress limit (Stat_Iso_Stress) and the statistically isotropic strain limit (Stat_Iso_Strain), respectively. The solid lines were obtained by integrating the microstructural specific heat shown in Fig. 1 and adding arbitrary constants to match each of the calculated limits.

strain energy changes during the CDW transitions and therefore a distribution of transition temperatures. This has the effect of smearing out the effective CDW transition temperature in the polycrystalline material. The elastic energy in the polycrystalline microstructure depends on the thermal history of the material. It is likely that different results could be found with different heat treatments of the polycrystalline material.

\section{CONCLUSION}

Differential scanning calorimetry measurements on samples of polycrystalline and single-crystal $\alpha$-uranium having equal masses and composition showed a difference in heat capacities from 90 to $300 \mathrm{~K}$. This difference was attributed to elastic strains in the polycrystalline microstructure. The elastic energy changes with temperature owing to anisotropic thermal expansions of the individual crystallites. This "microstructural heat capacity" depends on the thermomechanical history of the sample, in part because the thermal expansions or contractions are sufficiently large to induce internal plastic deformations in the material. Neutron powder diffractometry was used to measure distributions of elastic strains along different crystalline axes of the polycrystalline sample. Using the known elastic constants of $\alpha$-uranium, the elastic energy in the microstructure was calculated from these measured strain distributions. The temperature dependence of this elastic energy was consistent with the microstructural heat capacity measured by calorimetry. Finally, it is discussed how elastic energies in the microstructure can account for the apparent absence of charge-density-wave transitions in polycrystalline uranium.

\section{ACKNOWLEDGMENTS}

M.E.M. is grateful for helpful discussions with T. M. Holden. The Manuel Lujan, Jr. Neutron Scattering Center is funded by the U.S. Department of Energy, Office of Basic Energy Sciences-Materials Science, under Contract No. W-7405-ENG-36 with the University of California. Additional support was provided by the U.S. Department of Energy under Contract No. DE-FG03-96ER45572 and other programs.
${ }^{1}$ G. H. Lander, E. S. Fisher, and S. D. Bader, Adv. Phys. 43, 1 (1994).

${ }^{2}$ R. O. A. Hall, Inst. Phys. Conf. Ser. 27, 60 (1978).

${ }^{3}$ L. J. Nagel, B. Fultz, J. L. Robertson, and S. Spooner, Phys. Rev. B 55, 2903 (1997).

${ }^{4}$ M. E. Manley, B. Fultz, and L. J. Nagel, Philos. Mag. B 80, 1167 (2000).

${ }^{5}$ M. E. Manley, B. Fultz, R. J. McQueeney, C. M. Brown, W. L. Hults, J. L. Smith, D. J. Thoma, R. Osborn, and J. L. Robertson, Phys. Rev. Lett. 86, 3076 (2001).

${ }^{6}$ C. C. McPheeters, E. C. Gay, P. J. Karell, and J. P. Ackerman, JOM 49, 7 (1997).

${ }^{7}$ L. Anthony, J. K. Okamoto, and B. Fultz, Phys. Rev. Lett. 70, 1128 (1993).

${ }^{8}$ J. F. Nye, Physical Properties of Crystals: Their Representation by Tensors and Matrices (Oxford Science Publications, New York, 1989), p. 137.

${ }^{9}$ P. W. Stephens, J. Appl. Crystallogr. 32, 281 (1999).
${ }^{10}$ A. C. Larson and R. B. Von Dreele, GSAS-General Structure Analysis System, Los Alamos National Laboratory Report No. LAUR 86-748, 1986 (unpublished).

${ }^{11}$ A. Le Bail, H. Duroy, and J. L. Fourquet, Mater. Res. Bull. 23, 447 (1988).

${ }^{12}$ E. S. Fisher, J. Nucl. Mater. 18, 39 (1966).

${ }^{13}$ A. R. Stokes and A. J. C. Wilson, Phys. Soc. LVI, 3 (1944).

${ }^{14}$ E. S. Fisher and H. J. McSkimin, J. Appl. Phys. 29, 1473 (1958); Phys. Rev. 124, 67 (1961).

${ }^{15}$ C. S. Barrett, M. H. Mueller, and R. L. Hittermann, Phys. Rev. 129, 625 (1963).

${ }^{16}$ J. Crangle and J. Temporal, J. Phys. F: Met. Phys. 3, 1097 (1973).

${ }^{17}$ J. C. Lashley, B. E. Lang, J. Boerio-Goates, V. G. Woodfield, G. M. Schmiedeshoff, E. C. Gay, C. C. McPheeters, D. J. Thoma, and W. L. Hults, Phys. Rev. B 63, 224510 (2001).

${ }^{18}$ D. A. Tindall and M. O. Steinitz, J. Phys. F: Met. Phys. 14, 1131 (1984). 